\title{
Diversificación y autonomía en la política exterior latinoamericana*
}

\author{
FABIO FORERO**
}

Artículo recibido: 9 de octubre de 2014
Artículo aprobado: 4 de mayo de 2015

Doi: dx.doi.org/10.12804/desafios27.2.2015.09

Para citar este artículo: Forero, F. (2015). Diversificación y autonomía en la política exterior latinoamericana. Desafios, 27(II), 291-322. Doi: dx.doi.org/10.12804/desafios27.2.2015.09

\section{Resumen}

Este artículo indaga sobre el tratamiento que la literatura teórica ha dado al objetivo de diversificar la politica exterior. Para tal efecto, se proponen tres preguntas esenciales: ¿qué es?, ¿para qué sirve? y ¿qué factores la producen? Las respuestas ofrecidas por quienes abordan este tema son diversas y ambiguas. No obstante, el propósito central es hacer explicito el vinculo frecuentemente asumido entre diversificación y autonomía, asi como profundizar sobre cómo opera esta relación en la literatura sobre politica exterior latinoamericana.

Palabras clave: diversificación, política exterior latinoamericana, autonomía.

\footnotetext{
* Una versión preliminar de este artículo fue presentada en el Tercer Congreso de la Red Colombiana de Relaciones Internacionales (Redintercol), realizado en la Universidad Eafit de Medellín, el 27 y 28 de febrero de 2014.

** Estudiante de Doctorado en Ciencia Política, Universidad de los Andes. Correo electrónico: fa.forero10@uniandes.edu.co
} 


\title{
Autonomy and Foreign Policy Diversification in Latin America
}

\begin{abstract}
This article delves into the analysis that the theoretical literature bas given to the goal of foreign policy diversification. In order to do so, three key questions are asked: 1. what is foreign policy diversification?; 2. what is it used for?; and 3. what factors produce it? The answers offered by authors who address this issue are diverse and ambiguous. However, the main purpose of the article is to bighlight the often-assumed link between diversification and autonomy, and elaborate on how this relationship works.
\end{abstract}

Keywords: diversification, Latin American foreign policy, autonomy.

\section{Diversificação e Autonomia na política exterior latino-americana}

\begin{abstract}
Resumo
Este artigo indaga sobre o tratamento que a literatura teórica tem dado ao objetivo de diversificar a política exterior. Para tal efeito, propõem-se três perguntas essenciais: o que é?, para que serve?, e que fatores a produzem? As respostas oferecidas por quem abordam este tema são diversas e ambiguas. No entanto, o propósito central é fazer explicito o vínculo frequentemente assumido entre diversificação e autonomia, assim como aprofundar sobre como opera esta relação na literatura sobre politica exterior latino-americana.
\end{abstract}

Palavras-chave: Diversificação, politica exterior latino-americana, autonomia.

\section{Introducción}

La discusión sobre diversificación de la política exterior latinoamericana se inserta en un debate teórico más amplio, relacionado con el diseño de una inserción internacional enfocada a superar la condición dependiente que enfrentan algunos países. Esta condición podría ser entendida como la centralización de acciones externas, en uno 
o pocos actores, que limita las posibilidades de un comportamiento internacional más activo y autónomo. ${ }^{1}$

En la actualidad, es posible detectar una variedad de países que tienen interés en revertir dicho condicionamiento. Tal como lo refleja la discusión académica, este objetivo ha estado presente en países del Sudeste Asiático, como Indonesia, Filipinas, Malasia, Singapur, Tailandia y Vietnam (Goh, 2007, p. 183); en países de Asia Central, como Kazakistán, Kirgistán, Tayikistán, Turkmenistán y Uzbekistán (Tolipov, 2007); y en países latinoamericanos, como Argentina (Rubiolo, 2012), Brasil (Vigevani y Cepaluni, 2007), Chile (Faust, 2004), Colombia, México (Faust, 2002; Garza Elizondo, 1996), Perú (Olano, 2012) y Venezuela ('Tokatlian, 2011).

En una revisión preliminar general, ${ }^{2}$ se encuentra que este objetivo está presente en países cuyo interés consiste en aumentar su rango de

1 Agradezco a los evaluadores su comentario sobre la importancia de diferenciar la relación entre autonomía, dependencia e interdependencia. Esta diferenciación es posible encontrarla en el concepto de interdependencia desarrollado por Keohane y Nye (1988). Este establece que la interdependencia se refiere a situaciones caracterizadas por efectos de costo recíproco que resultan de los intercambios entre países. Así, la dependencia es "un estado en que se es determinado o significativamente afectado por fuerzas externas" (1988, p. 23). Bajo esta perspectiva, las relaciones interdependientes siempre implicarán costos, porque reduce la autonomía. Pero señala que es imposible determinar a priori si los beneficios de una relación serán mutuos o mayores a sus costos. Si bien Keohane (1988) no dialoga con la teoría de la dependencia, esta última se ha caracterizado por mostrar los costos asimétricos en que incurren los países periféricos al intercambiar con países del centro. Desde esta lógica, se entiende que, en situaciones de efectos recíprocos entre países, a mayor costo menor autonomía (Keohane y Nye 1988).

2 A partir de una revisión de los objetivos de política exterior disponibles en una amplia muestra de páginas web de las cancillerías y presidencias de países en distintas regiones del mundo con diferentes características en términos de tamaño y nivel de desarrollo, se ha detectado que el objetivo de la diversificación no está presente en países grandes ni en los países menos desarrollados, ya que los primeros cuentan con relaciones diversificadas, y los segundos tienen políticas exteriores muy limitadas que les impiden buscar mayor posicionamiento internacional. De esta forma, se ha identificado que el problema de la diversificación es posible circunscribirlo a países pequeños y medianos en proceso de consolidación estatal con niveles medios y altos de desarrollo económico. El término diversificación en países grandes y desarrollados es usado para referirse a los objetivos de ampliación de fuentes energéticas y mayor acceso a recursos naturales, connotación diferente a aquellas que se le dan al término en los países del Sur global. 
acción y margen de maniobra internacional. En el caso de los países de América Latina, independientemente de su tamaño y relación con los Estados Unidos, Tokatlian (2011) sostiene que hay una tendencia proveniente del pasado que está dirigida a adelantar políticas de diversificación para lograr mayor autonomía y desarrollo nacional.

Pero ¿a qué se refiere la diversificación de la política exterior latinoamericana? Este es un término sobre el que ha habido poca discusión en la literatura especializada y tampoco suscita grandes controversias en la práctica, ya que se supone positiva. A pesar de su importancia, padece una relativa invisibilidad académica en el estudio de la política exterior. Son pocos los trabajos existentes que se refieren a este asunto de forma específica. Por lo tanto, las respuestas ofrecidas por la literatura a los interrogantes ¿qué es?, ¿qué factores la producen? y ¿para qué sirve? son inconclusas y ambiguas. Por consiguiente, el propósito de este artículo es mostrar el vínculo entre diversificación y búsqueda de mayor autonomía, al mismo tiempo que profundiza sobre la forma cómo opera esta relación. En la primera parte, se presentan diferentes definiciones dadas a este término. En la segunda, se exponen las explicaciones relacionadas con los factores que producen este fenómeno. Y en la última sección, se explora el origen del concepto en el legado de dependencia que condiciona a los países latinoamericanos, y se muestra cómo opera esta relación entre diversificación y autonomía.

\section{De la noción de diversificación de exportaciones a la estrategia 'omni-enmeshment'}

Los trabajos existentes nos ofrecen tres formas de pensar este término. En primer lugar, un grupo de autores lo entiende como una política pública, mediante la cual es posible diseñar y ejecutar un conjunto de acciones para diversificar. Esta aproximación no contempla ni profundiza en las restricciones que impone el sistema internacional. Es decir, se trata en mayor medida de una definición doméstica. En cambio, otro conjunto lo identifica con un tipo de comportamiento determinado en la estructura de distribución de poder. En tercer lugar, otros académicos lo perciben como un modelo de inserción global, o medio para alcanzar mayor autonomía. 


\subsection{Instrumento de política pública}

Un conjunto de estudios se ha concentrado en la explicación de la diversificación de las exportaciones con herramientas teóricas provenientes de la literatura sobre comercio internacional (Ali, Alwang \& Siege, 1991; Gutiérrez de Piñeres \& Ferrantino, 1997; Al-Marhubi, 2000; Rodrik, 2005; Ben-Hammouda, Karingi, Njuguna \& SadniJallab, 2006; Agosin, 2007; Amurgo-Pacheco \& Pierola, 2007; Mejía, 2011). Estos trabajos la conciben de dos formas: una que se refiere al incremento de las exportaciones hacia distintos mercados (Ali et al., 1991) y otra que es entendida como el crecimiento de la producción en nuevos sectores económicos (Berthelemy y Chauvin, 2000). Así mismo, puede tener dos dimensiones: una horizontal y otra vertical (Samen, 2010). La horizontal comprende la adición de nuevos productos a la canasta exportadora de un país, mientras que la vertical se refiere al movimiento del sector económico primario hacia la industria y/o suministro de servicios (Samen, 2010). Por consiguiente, solo aquella definición en el primer sentido aquí expresado es consecuente con el concepto de ampliación de las relaciones exteriores, ya que la segunda concepción no necesariamente implica generación de nuevos socios. Es decir, es posible lograr un incremento de las exportaciones en diferentes sectores productivos que estén concentrados en un solo país de destino.

En el ámbito de la política exterior, un grupo de autores se apoya en la noción de diversificación económica para explicar este concepto como un aumento de socios económicos y políticos (Wilhelmy y Lazo, 1997; Faust y Mols, 1998; Faust, 2002, 2004; Olivet, 2005; Baroni y Rubiolo, 2013; Rubiolo, 2011, 2012; Fernández de Soto y Pineda, 2012; Protsenko, 2013). Por lo tanto, esta noción es pensada como una política pública que obedece a los esfuerzos y recursos domésticos desplegados por un país para incrementar sus relaciones internacionales.

Sin embargo, es importante diferenciar estos dos enfoques. En primer lugar, la creación de nuevos mercados no necesariamente implica el crecimiento del radio de acción de la política exterior, puesto que esta 
incorpora elementos adicionales a las exportaciones. Por ejemplo, comprende acciones externas, tales como la negociación de acuerdos y tratados, el desarrollo de agendas de alto nivel, la promoción de actividades culturales en el exterior, el aumento de los flujos de inversión extranjera, la coordinación de políticas en el plano multilateral, la cooperación ofrecida y recibida, el establecimiento de relaciones diplomáticas y la apertura de embajadas. En contraste, la aproximación del comercio internacional se refiere estrictamente a estrategias de crecimiento económico, mientras que la otra busca explicar el comportamiento internacional de un país.

Una vez ha sido aclarada esta diferencia, es posible preguntar ¿cómo ha sido entendida la diversificación de la política exterior latinoamericana? Jorg Faust la define en dos dimensiones. Una primera dimensión se refiere a la incorporación de nuevas áreas de política pública y la segunda a la ampliación de nexos con nuevos países o regiones (Maihold, 2011, p. 207). De esta manera, se entiende como "el intento por parte de un Estado de ampliar sus relaciones con regiones con las que hasta el momento solo se mantenían contactos rudimentarios, a través del diseño de nuevos campos de política o de la modificación de los ya existentes" (Faust y Mols, 1998, p. 10). Por su parte, Rubiolo (2010) \& Olivet (2005) señalan que este es un instrumento de inserción en la economía mundial para evitar ser periféricos y tener mayor voz a nivel internacional.

Es importante resaltar que estos trabajos no acuden a mecanismos de medición sofisticados, como sí lo hace la literatura sobre la diversificación de exportaciones. En el ámbito de la política exterior, los autores acuden a distintos indicadores para identificar esta política pública, los cuales pueden agruparse en tres dimensiones: aumento de relaciones bilaterales (establecimiento de relaciones diplomáticas por país, tratados y acuerdos, apertura o cierre de embajadas y consulados, tamaño de personal por país, cooperación técnica y financiera ofrecida y recibida, centros de difusión cultural, oficinas comerciales, mecanismos de diálogo político, visitas de funcionarios, presupuesto), incremento de comercio bilateral (destino de las exportaciones y origen de las importaciones) y mayor participación en organismos 
multilaterales (grupos de concertación a los que pertenece, cumbres interregionales, votación en la ONU, organizaciones regionales y globales a las que pertenece).

Ahora bien, ningún estudio acude al mismo conjunto de indicadores para contrastarlos en el tiempo o entre distintos países. Es decir, no existe un mecanismo único de medición que permita rastrear la ejecución de esta política pública en términos comparativos. Por consiguiente, es erróneamente entendida en términos absolutos como una característica que se tiene o no se tiene. En consecuencia, se dificulta la identificación de patrones de comportamiento a lo largo del tiempo que permita explicar períodos de expansión o contracción, e imposibilita la comparación de resultados entre diferentes países. Avanzar en la creación de mecanismos de medición podría facilitar la identificación y comparación entre distintos países.

\subsection{Tipo de comportamiento internacional}

Otro enfoque es el utilizado por Kal Holsti (1982). Estos autores realizan ocho estudios de caso sobre seis países durante el período de la Guerra Fría (tres sobre China, Bhutan, Tanzania, Canadá, Chile y Burma), con el fin de tener una muestra que reúna países desarrollados y no desarrollados, grandes y pequeños, autoritarios y democráticos. Su objetivo principal consiste en explicar la 'restructuración' de la política exterior a partir de las características de los distintos países. Por esta razón, se analizan períodos cortos de tiempo donde se reflejan cambios dramáticos y tiene como propósito central identificar los factores que inciden para que un país 'reajuste' su orientación externa.

Holsti (1982) construye una tipología de diferentes comportamientos que puede seguir un país, entre los cuales se encuentran: aislamiento, autosuficiencia, dependencia y no alineación-diversificación. Bajo este enfoque, este concepto es entendido como un tipo de comportamiento internacional. Aquello interesante es lo que nos permite trazar un continuo entre dependencia y diversificación. Además, introduce una dimensión de cambio en la política exterior. En otras 
palabras, explican el cambio de un tipo de comportamiento a otro en la política exterior de los países que estudian. Sin embargo, vale la pena preguntarse ¿en qué medida es posible decir que un país es diversificado? Es decir, este trabajo piensa el tipo de comportamiento internacional de un país como un resultado que es producido a través del desarrollo de un conjunto de acciones externas.

\subsection{Modelo de inserción internacional}

Un tercer grupo de autores considera que la diversificación es un modelo de inserción internacional que permite incrementar la autonomía y superar la condición de dependencia (Jaguaribe, 1979; Puig, 1980; Drekonja, 1993; Russell \& Tokatlian, 2010). Al respecto, Gerhard Drekonja (1993) la asocia a una estrategia de política exterior de alto perfil realizada por países que son más activos a nivel internacional (1993, p. 17). Otros autores, como Tullo Vigevani y Gabriel Cepaluni (2007), van más allá. La definen como la adhesión a normas y principios internacionales, a través de alianzas sur-sur y acuerdos con socios no tradicionales, para reducir asimetrías con países poderosos y lograr mayores niveles de autonomía. Cabe señalar que en estos trabajos no existe un interés por medir o identificar la diversificación, debido a que no constituye el eje central de lo que desean explicar.

Por su parte, Farkhod Tolipov (2007) también proporciona una definición. Este autor analiza el caso de seis países postsoviéticos ubicados en Asia Central (Kazajistán, Kirguistán, Tayikistán, Turkmenistán y Uzbekistán). En este trabajo, entiende la diversificación como una orientación de la política exterior que tiene una doble connotación: una económica y otra geopolítica. Al mismo tiempo, destaca que esta puede ser positiva o negativa. La primera tiende a evitar el enfoque de suma cero y es de carácter inclusivo, mientras que la segunda se refiere a la revitalización del balance de poder en relaciones internacionales y a los juegos de suma cero entre los grandes poderes a expensas de los países más pequeños. Mientras la definición de Holsti (1982) se suscribe al comportamiento de alineación o no, esta definición se refiere a un modelo de inserción internacional. Es decir, comprende un conjunto de acciones basadas en la búsqueda de aliados para 
incrementar la autonomía en relación con el control de los recursos naturales, en un contexto de vulnerabilidad frente a factores sistémicos de balance de poder entre potencias.

Por su parte, Evelyn Goh (2008) crea una noción de diversificación asociada al modelo omni-enmeshment que desarrollan los países del Sudeste Asiático para insertarse en el mundo. Esta consiste en comprometerse con otro país o grupo de países para profundizar vínculos en la sociedad regional o internacional envolviéndolo en una red de intercambios a lo largo del tiempo que facilite la integración de los intereses en el largo plazo. En este proceso, la identidad de un país puede ser alterada para garantizar la integridad y orden del sistema. Es bajo esta noción más amplia que se incorpora el concepto de diversificación estratégica, el cual se refiere a la implementación práctica de este modelo en un rango de asuntos que promueven los objetivos prioritarios de un país y lo empoderan frente a otro.

Este tipo de estrategia es ejecutada en escala multinivel. Un primer nivel es aquel sostenido con los países más poderosos en la región, otro nivel en el ámbito multilateral y un tercer nivel en el plano bilateral. De esta manera, según Goh (2008) los países del Sudeste Asiático han adoptado una noción de diversificación amplia, multidireccional y entramada (p. 121). Es relevante destacar de esta definición la posibilidad que abre para comprender el omni-enmeshment a largo plazo como un proceso que conlleva la alteración de factores identitarios en los patrones de interacción entre países a través de la implementación de la diversificación estratégica. Cabe subrayar que Goh no desarrolla este enfoque ni ha sido incorporado en los estudios existentes, pero que podría resultar como una aproximación innovadora al tema.

A partir de lo anterior, es posible señalar que existen dos grupos de trabajos que tocan este tema. Un primer grupo entiende la diversificación como un fin. Estos autores se proponen explicar los factores que producen este resultado de política exterior. El segundo grupo de trabajos la asimila como un medio o instrumento que es activado para la consecución de autonomía. 


\section{Factores que producen la diversificación de la política exterior}

En relación con los factores que producen la diversificación, las explicaciones existentes son ambiguas y poco exploradas en forma sistemática. Solamente el estudio de Faust (2004) tiene como propósito explicar los factores que producen políticas de diversificación más exitosas. En este sentido, vale la pena preguntarse ¿los factores determinantes se ubican en el nivel sistémico o en el nivel doméstico? y ¿cuál explicación tiene mayor poder para abarcar un mayor número de casos?

La mayor parte de los autores que han estudiado el fenómeno de la diversificación no diferencian el nivel de influencia de los factores domésticos respecto de los sistémicos, ni se prioriza alguno sobre otro. Esto conduce a explicaciones que describen una interacción entre los dos niveles sin proporcionar claridad sobre aquello que conduce a un país o grupo de naciones hacia una mayor o menor diversificación.

Faust (2002 y 2004) es el único que avanza en esta dirección al realizar un estudio de caso para México y otro para Chile. Al comparar estos dos casos, establece que el comportamiento de las élites ha sido fundamental. En el caso de México, las élites han impedido el desarrollo de una política exterior exitosa en la ampliación de la relación con Asia. En cambio, en el caso de Chile, las élites han marcado la diferencia, debido a que han construido redes de organizaciones no estatales para influenciar la política de ampliación de relaciones con Asia. Ahora bien, esta comparación tiene un problema. Este autor asume las características del sistema internacional como una constante. Es decir, considera que el contexto internacional influencia de igual manera a los dos países, poniendo un mayor énfasis explicativo sobre los aspectos domésticos.

Para Holsti (1982), no es posible generalizar una explicación sobre los factores que producen la restructuración de la política exterior de un país a partir de sus características. No obstante, en los diferentes 
casos pueden encontrarse explicaciones influenciadas tanto por el nivel individual como por el sistémico. En el nivel individual, el caso de Chile y Tanzania se atribuyen a motivaciones de los líderes por superar condiciones de dependencia. En el nivel sistémico, los casos de China, Bhutan y Burma se explican por condiciones de dependencia económica y balance de poder frente a los potencias en la región.

En el caso de los países del Sudeste Asiático, Goh (2008) indica que factores sistémicos como el ascenso de China como potencia y la preponderancia de los Estados Unidos llevan a estos países a crear estrategias que les permitan mantener su autonomía y, al mismo tiempo, evitar la dependencia en grandes potencias, bajo un enfoque de balance frente a diferentes amenazas.

Por último, Gomes Saraiva (2011) analiza el papel de la burocracia en la diplomacia brasilera. Esta autora indica que existen dos corrientes en el interior de la cancillería de ese país, una autonomista y otra liberal. Según esta autora, los ajustes que se han dado en el modelo de inserción internacional de Brasil se deben al mayor posicionamiento de los autonomistas durante los últimos diez años. Por consiguiente, otro de los posibles factores que pueden explicar la diversificación de las relaciones exteriores proviene del nivel burocrático en el ámbito doméstico.

\section{Papel e importancia de la diversificación de la política exterior}

En el ámbito de la política exterior latinoamericana, la preocupación por la autonomía ha estado presente de manera permanente en el debate (Giacalone, 2012). En este grupo de trabajos, se hace referencia a la diversificación, pero se entiende de manera implícita como algo positivo para la autonomía. Tampoco existen estudios que hayan profundizado en esta relación. Por lo tanto, el propósito central de este artículo gravita alrededor de la pregunta ¿por qué orientar la política exterior hacia la diversificación? Los autores que han trabajado este tema parten de la existencia de una necesidad de redefinir 
o reorientar la política exterior para superar las limitaciones que impone la centralidad en uno o pocos interlocutores. Este aspecto se encuentra implícito en la mayoría de estudios, pero es un supuesto que merece ser explorado en mayor profundidad para comprender el papel e importancia que tiene.

\subsection{Legado de dependencia económica y política}

Vale la pena remontarse a las ideas de Raúl Prebisch para comprender por qué un país decide incrementar sus socios. Estas ideas se convirtieron en iniciativas de 'desarrollo desde dentro' y en una pretensión de los países en desarrollo por modificar la estructura económica internacional. Además, tuvieron un impacto generalizado en el diseño de estrategias de desarrollo en América Latina (Pollock, Kerner \& Love, 2001). Siguiendo a Ocampo (2008), pueden destacarse tres planteamientos centrales en las nociones de Prebisch. Primero, la existencia de asimetrías internacionales entre el centro y la periferia. Segundo, la necesidad de adoptar estrategias activas de 'desarrollo desde dentro'. Y, tercero, el papel de la integración regional en el modelo de desarrollo (2001, p. 27).

Estas asimetrías provienen de dinámicas de intercambio desigual entre estructuras productivas diferenciadas geográficamente. La estructura productiva del 'centro' es diversificada y especializada en las exportaciones de bienes de alta elasticidad-ingreso. En cambio, la estructura productiva de la 'periferia' es poco diversificada y se concentra en la exportación de bienes de baja elasticidad de ingreso. Para estos autores, los precios de los bienes que se producen en el 'centro' tienen una tendencia al alza, mientras que los precios de los bienes producidos en la 'periferia' siguen una tendencia decreciente (Ocampo, 2008).

Posteriormente, estas concepciones fueron profundizadas por Theotonio dos Santos, André Gunder Frank, Fernando Cardoso, Enzo Faletto y Osvaldo Sunkel en la elaboración de la teoría de la dependencia. Estos académicos fueron propulsores del instrumento de sustitución de importaciones y de los esquemas de integración regional que tenían como propósito modificar la estructura productiva 
dependiente de los países del 'centro' y el lugar de los países 'periféricos’ en la economía mundial.

Las ideas de la Cepal y de la Escuela de la Dependencia no solo tuvieron un impacto en la formulación de los modelos de desarrollo, sino también en la concepción de la política exterior. Al respecto, Helio Jaguaribe (1979) concebía un sistema internacional jerárquico condicionado por el sistema productivo. En el período de la Guerra Fría, según este autor, la estructura de poder se asemejaba a un sistema imperial, dividido en dos áreas de influencia hegemónica. Por lo tanto, los países se encontraban estratificados de acuerdo con su capacidad de autodeterminación: primacía general, primacía regional, autonomía y dependencia.

En este esquema, los Estados Unidos y la Unión Soviética se ubican en el nivel de primacía general. Esta se caracteriza por la inviolabilidad y ejercicio de presencia mundial generalizada. En el grado de primacía regional, se sitúa a China, por tener capacidad para proteger su territorio, ejercer hegemonía sobre áreas determinadas, tener presencia preponderante en otras y restricciones en otras (1979, p. 92). En el nivel de la autonomía, está Europa Occidental y Japón. Este nivel lo entiende como una condición bajo la cual un país no es capaz de garantizar la inviolabilidad de su territorio, pero dispone de medios para imponer penalidades materiales y morales a un eventual agresor, al mismo tiempo que tiene un margen amplio de autodeterminación en la conducción de sus asuntos internos y capacidad de actuación internacional.

En el último estrato, se encuentra el nivel de la dependencia. Aquí, Jaguaribe (1979) ubica al resto de los países del mundo que se caracterizan por poseer una condición nominal de soberanía, tener órganos de gobierno propios y estar acreditados como interlocutores independientes ante otros actores internacionales; pero que dependen de decisiones y factores externos que provienen de países con primacía general o regional. Bajo esta perspectiva, se crea una relación entre la capacidad de autodeterminación y el tipo de inserción internacional a la que un país puede aspirar. 
Por otro lado, Juan Carlos Puig (1980) parte de esta concepción y ofrece elementos adicionales sobre el papel de las élites en la condición de dependencia. Al respecto, sugiere una disfuncionalidad de las élites que no aprecian por ceguera o por conveniencia las posibilidades de proyectos autonomizantes. En ese sentido, juegan un papel de dominantes-dominados, ya que transfieren los requerimientos del dominante externo actuando por su cuenta y orden, al mismo tiempo que afianzan su dominación interna subordinada (Puig, 1980, p. 149). En consecuencia, para este autor, el tránsito de la dependencia hacia la autonomía reside en las actitudes que adoptan o pueden adoptar las élites.

En esta misma línea, otros autores, como Gunder Frank, Cardoso y Faletto, sostienen que la influencia de la dependencia comercial se traduce en subordinación política. Estos autores manifiestan que la dependencia se mantiene por la existencia de intereses comunes y coaliciones entre las clases gobernantes del centro y de la periferia. Los grupos de poder en la periferia someten sus intereses al centro con el propósito de legitimarse a nivel interno (Tickner, 2002, p. 51).

La influencia de la dependencia económica en el plano político también fue trabajada por autores que no pertenecen a la escuela de la dependencia latinoamericana. Bruce Moon (1983), Neil Richardson \& Charles Kegley $(1980)^{3}$ han investigado esta relación. Al respecto, se preguntan por la forma como los países dominantes ejercen influencia en materia de política exterior sobre países dependientes (Moon, 1983, p. 316; Richardson \& Kegley, 1980). En efecto, muestran los nexos entre la política económica y la política exterior a partir de los flujos de comercio internacional y las posiciones en los foros multilaterales. Al observar los patrones de votación en las Naciones Unidas, encuentran una alta cooptación o alineación de los países periféricos con los intereses multilaterales de los Estados Unidos. Por lo tanto, la política internacional es entendida como un 'intercambio' o pago

\footnotetext{
Estos autores trabajan la noción de dependencia, pero no hacen parte de la escuela dependentista latinoamericana.
} 
por el mantenimiento de beneficios que son derivados de lazos económicos con el país dominante (Richardson \& Kegley, 1980, p. 198).

Estas explicaciones nos permiten identificar tres razones esenciales que determinan la orientación de un país hacia la dependencia o hacia la diversificación, tales como la división internacional del trabajo, la visión de las élites de un país y la influencia de la dependencia económica en la cooptación política de la acción internacional.

\subsection{Efectos de la dependencia}

El legado de dependencia ha ocasionado que la relación con los Estados Unidos sea el eje principal en la orientación de la política exterior para la mayoría de los países débiles y dependientes que se ubican en su ámbito de influencia. En el caso de los países latinoamericanos, estos suscriben la relación con los Estados Unidos en un continuo entre dependencia y autonomía (Garza Elizondo, 1996; Sánchez, 2003; Bandeira, 2010; Escudé, 1995; Pardo \& Tokatlian, 1988; Blum, 2003). Por ejemplo, Fernando Garza Elizondo (1996) expresa que, en el caso de México, la estrategia de diversificación tiene una intención clara de disminuir la dependencia respecto a los Estados Unidos, pero, al mismo tiempo, señala que parece existir un fatalismo inevitable que lo condena a seguir el camino de una mayor vinculación y subordinación frente a la potencia hegemónica. ¿Qué explica esta situación? Según este autor, la inestabilidad política interna y los efectos sistémicos económicos reducen la autonomía y la soberanía frente a los Estados Unidos.

Por ello, la influencia de los países más poderosos y de la estructura internacional refuerzan la condición de dependencia y, a su vez, la debilidad del Estado. Así pues, la consolidación del Estado se convierte en un aspecto relevante frente a la motivación que tiene un país de orientar o no su política exterior hacia la diversificación.

La literatura sobre países débiles puede contribuir a explicar las particularidades de la inserción internacional en una condición dependiente. En primer lugar, se parte de la idea de una divergencia 
entre el modelo de Estado europeo y la realidad de los países débiles (Buzan, 1991; Jackson, 1993; Ayoob, 1995; Inayatullah, 1996; Kahler, 2000; Herbst 2000). Los procesos de consolidación del Estado en los países débiles no han seguido los mismos estadios de formación de los Estados europeos, debido a la existencia de un contexto internacional que protege la existencia legal de Estados poscoloniales sin que necesariamente se encuentren cohesionados internamente (Ayoob, 1995, p. 81).

En ese sentido, como lo indica Arlene Tickner (2003), la literatura de Estados débiles examina las variables internas que hacen particular su proceso de consolidación estatal, siendo estas: la legitimidad, el nacionalismo, el ejercicio de la soberanía y la autonomía. Los aportes de la teoría de la dependencia proporcionados por autores como Cardoso y Faletto le adjudican las disfunciones del Estado a la lógica del capitalismo global. Para estos autores, la dependencia es creada y recreada a través de la división internacional del trabajo y se manifiesta en diversas formas de dominación económica, cultural, política y social (Tickner, 2003, p. 317). Tomando como base este planteamiento, la debilidad del Estado es un producto histórico de la inserción internacional de los países dependientes que condiciona su soberanía y autonomía (Tickner, 2003).

Por ende, la reorientación de la política exterior de un país hacia la diversificación puede estar motivada en el deseo de modificar su inserción internacional dependiente, ya que esta condiciona las posibilidades de un mayor fortalecimiento del Estado. En virtud de lo anterior, el nivel precario de consolidación del Estado y su efecto sobre el ejercicio de la soberanía y la autonomía de una nación son aspectos fundamentales para comprender el comportamiento internacional de países débiles. Es por esta razón por la que es importante profundizar en el problema de la soberanía y la autonomía.

\subsection{Diversificación, consolidación estatal y autonomía}

La autonomía es una variable que en los países débiles resulta fundamental para comprender los procesos de diversificación de las 
relaciones exteriores. Distintos enfoques de política exterior latinoamericana identifican una conexión entre diversificación y autonomía, la cual establece que al aumentar una también se incrementa la otra, y, por lo tanto, se refuerza el proceso de consolidación estatal (Jaguaribe, 1979; Puig, 1980; Drekonja, 1993; Cepeda, 1983; Pardo \& Tokatlian, 1988; Tokatlian, 1996; Vigevani \& Cepaluni, 2007; Russell \& Tokatlian, 2010). En consecuencia, se sostiene que el tipo de autonomía que es capaz de ejercer un país influye de forma determinante sobre su modelo de inserción internacional, sea este: aislamiento, dependencia o diversificación. La literatura nos permite identificar distintos tipos de autonomía: periférica (Jaguaribe, 1979; Puig, 1980; Drekonja, 1993), asociada (Escudé, 1995), ${ }^{4}$ relacional (Russell \& Tokatlian, 2010; Vigevani \& Cepaluni, 2007), autonomía a través de la diversificación (Vigevani \& Cepaluni, 2007) y confrontacional (Puig, 1980). Cabe señalar que no se plantea un desarrollo o gradación de un extremo a otro, sino que el tipo de autonomía está asociado a las capacidades del Estado. En ese sentido, el tipo de autonomía no es aquella que se escoge, sino aquella que es posible ejercer.

\subsubsection{Apuntes sobre la experiencia latinoamericana}

La autonomía periférica (Drekonja, 1993) o heterodoxa (Puig, 1980) es aquella mediante la cual los grupos de poder en el interior de un país periférico tratan de aprovechar las debilidades y errores del centro del poder mundial, aceptan el liderazgo de las potencias dominantes y, solamente en cuestiones cruciales que son determinadas por el país más poderoso, optarán por responder a las aspiraciones del centro. En la región, durante la década de los setenta y la década de los ochenta, solo fue posible desarrollar una autonomía parcial en el campo de una política de comercio exterior diversificada (Drekonja, 1993, p. 16).

En consecuencia, indica Drekonja (1993), las relaciones con los Estados Unidos se debilitaron. Inversamente, se fortalecieron las relacio-

\footnotetext{
4 Es importante anotar que, para este autor, la secuencia es distinta, porque entiende que el desarrollo económico es aquel que posibilita la consolidación del Estado y el resultado de esos dos procesos es lo que permite acceder a la autonomía.
} 
nes político-comerciales con Europa, Japón y los Estados árabes. La relación con Europa fue asimilada como una cooperación preferencial entre las dos regiones dentro del sistema americano para obtener un margen de maniobra parcial. En otras palabras,

esto significó para la nueva práctica de la política exterior latinoamericana de los años setenta un ensanchamiento geográfico, mayor diversificación diplomática, pluralismo ideológico, acercamiento a los países no alineados, convenios sobre materias primas, realización de iniciativas sobre política tecnológica, establecimiento de firmas multinacionales para defenderse de los consorcios trasnacionales y legislación sobre capital extranjero... así como la reducción de conflictos intrazonales. (Drekonja, 1993, p. 17).

Tomando como base lo anterior, este autor sugiere un vínculo directo entre un modelo de inserción internacional diversificado y autonomía, el cual se asimila como instrumento para acceder a mayor control y como una característica de aquellos países latinoamericanos que exhiben un 'alto perfil' en la conducción de su política exterior. Por su parte, Fernando Cepeda (1983) afirma que una política exterior que aspire a tener mayor independencia debe contar con respaldo material en dos recursos: "Diversificación del comercio exterior para que la economía periférica sea cada vez menos dependiente de un solo centro hegemónico y mantenimiento de cuantías importantes de reservas internacionales en relación con el promedio del mismo país y de los pares regionales" (Tokatlian, 1993, p. 32).

Con el paso del tiempo, varios gobiernos latinoamericanos mantuvieron la tendencia de ampliar las relaciones comerciales con nuevos socios. Incluso, estos esfuerzos se circunscriben en el ejercicio de una autonomía periférica. $\mathrm{Al}$ respecto, distintos autores se han propuesto evaluar los acercamientos bilaterales en términos políticos, comerciales y de inversión entre Latinoamérica y otras regiones. En este sentido, varios de ellos coinciden en subrayar que existe una tendencia divergente entre los esfuerzos de ampliación de relaciones comerciales con los esfuerzos de ampliación de relaciones políticas. Por un lado, se subraya la sobredimensión de los aspectos comerciales y, por el 
otro, la desatención de los asuntos políticos (Wilhelmy \& Lazo, 1997; Faust \& Mols, 1998; Cardona, 2001; Pardo, 2001; Faust, 2002, 2004; Rubiolo, 2011; Barbosa, Posada \& Serrano, 2011; Rubiolo, 2012; Fernández de Soto \& Pineda, 2012; Olano, 2012; Vargas, Sosa \& Rodríguez Río, 2012; Baroni \& Rubiolo, 2013).

Por ejemplo, Guillermo Fernández de Soto \& Saúl Pineda (2012) estimaron que las exportaciones de América Latina y el Caribe crecieron en la última década de 50.000 millones de dólares a 150.000 millones de dólares en 2011 (p. 116), siendo China, Japón y Corea del Sur los principales mercados de destino en Asia (p. 117). Estos estudios muestran diferentes grados de profundidad en la ejecución de estrategias de diversificación económica por parte de distintos países latinoamericanos, como Chile, México, Perú, Colombia y Argentina. Destacándose Chile y Brasil en materia de diversificación económica, en la medida que han logrado transformar la estructura de destino de exportaciones y han cambiado como principal socio comercial a los Estados Unidos por China.

Es más, como lo señala Faust (2004), en el caso de Chile se ha dado un fenómeno de economicización de la política exterior, donde los esfuerzos por ampliar los lazos políticos con otros países han sido más precarios (Faust, 2004). Por otro lado, en relación con el caso de Perú, Olano (2012) señala que este país ha avanzado mediante la negociación de acuerdos de libre comercio con un amplio número de países, pero la evaluación de resultados la circunscribe exclusivamente al plano económico, al mostrar que la ampliación de mercados no está produciendo una transformación en la estructura productiva del país y está reforzando una tendencia hacia un multilateralismo dependiente o dependencias múltiples que no necesariamente están diversificando el sector productivo, y, al contrario, está reforzando la dependencia de la estructura productiva en exportaciones primarias.

En otro orden de cosas, una visión más amplia es la de la autonomía relacional (Russell \& Tokatlian, 2010). Bajo esta perspectiva, la relación entre mayor diversificación y mayor autonomía se fortalece 
a través de una mayor participación en los asuntos globales. Este tipo de autonomía justifica la necesidad de ampliar y universalizar las relaciones externas de un país. $\mathrm{Al}$ mismo tiempo, presupone como requisito una mayor interacción, negociación y participación activa en la construcción de una gobernanza global. Así, la autonomía es redefinida como el poder requerido para participar e influenciar los asuntos mundiales. De esta manera, no compite con otros intereses nacionales, como seguridad y bienestar, pero debe ser concebida como un mecanismo para reducir asimetrías de poder a través de la participación activa. Además, la autonomía no debe pensarse en contraposición a los Estados Unidos, puesto que es posible la combinación entre mayor margen de maniobra y coincidencia con los intereses de este país (Russell \& Tokatlian, 2010, pp. 16-17).

Una noción en la misma dirección a la planteada por Roberto Russell y Juan Tokatlian (2010) es la autonomía por medio de la participación o integración (Vigevani \& Cepaluni, 2007; Vigevani Fernandes de Oliveira \& Simpson, 2007). Esta se entiende como la adhesión a los regímenes internacionales, pero sin perder el manejo de su política exterior. Paralelamente, tiene por objetivo influenciar la formulación de principios y reglas en el sistema internacional. Este fue el enfoque de inserción que ejecutó Brasil durante la década de los noventa. Durante este período, dicho país adoptó una agenda global proactiva y alineada con el liberalismo. La estrategia consistía en contribuir activamente en la elaboración de normas en el orden global y en el mantenimiento de un ambiente favorable para el desarrollo económico que le permitiese ganar mayor control sobre la solución de sus problemas internos. Este esquema lo implementó adoptando varias agendas y posiciones desde el nivel bilateral al mundial, buscando mercados y relaciones comerciales bajo el principio de no atarse a un solo socio, pero sin que significase el sacrificio de las buenas relaciones con los Estados Unidos. De esta forma, Brasil renovó su universalismo a través del concepto de autonomía y le apuntó a la diversificación de las relaciones exteriores para aumentar su credibilidad externa (Vigevani \& Cepaluni, 2007; Vigevani Fernandes de Oliveira \& Simpson, 2007). 
Posteriormente, dicen estos mismos autores, Brasil ejecutó la autonomía a través de la diversificación en los primeros años del siglo XXI (Vigevani \& Cepaluni, 2007). De esta manera, se entiende la ampliación de socios como un canal o instrumento de inserción internacional para ejercer mayor control sobre su comportamiento interno e internacional. Bajo esta perspectiva, Brasil buscaba ejercer la independencia frente a los países más poderosos, mediante el fortalecimiento de lazos con el Sur tanto en el nivel bilateral como en el nivel de las instituciones internacionales. Su objetivo consistía en lograr mayor poder en negociaciones internacionales y reducir asimetrías con los países más poderosos. Para tal efecto, Brasil amplió sus relaciones con países de habla portuguesa, incluyendo naciones africanas, y decidió avanzar en relaciones tanto económicas como políticas con nuevos socios.

Por otra parte, la autonomía confrontacional consiste en el desafío global y la ruptura del lazo de dependencia que une a un país periférico con el centro. Esta estrategia está vinculada con la viabilidad nacional y el funcionamiento del sistema internacional, en la medida en que estos factores posibilitan su ejercicio (Puig, 1980, p. 153). A partir de este enfoque, el modelo de inserción internacional también estará determinado por la permisibilidad internacional, la cual al mismo tiempo es influenciada por la capacidad económica y militar, la situación geopolítica de dicho país, sus relaciones con los demás países y las alianzas defensivas que establezca.

Para ilustrar, el caso de Venezuela es un ejemplo de autonomía confrontacional. Cameron Thies (2014) realiza un estudio del rol nacional que siguió la política exterior de Venezuela durante el período de mandato del presidente Hugo Chávez. Al respecto, señala que este país se caracterizó por ejercer un rol nacional revolucionario, liberador y antiamericano, a fin de buscar una mayor autonomía. Como lo señala Puig (1980), una ruptura requiere nuevas alianzas defensivas y relaciones internacionales con nuevos socios que le permitan al país dependiente estar en capacidad de desatender los intereses estratégicos del centro. Para tal efecto, Venezuela ha seguido esta estrate- 
gia, puesto que ha reorientado su política exterior y confrontado a los Estados Unidos mediante la construcción de alianzas con otras naciones, como China, Irán y Rusia, para hacer un contrapeso a la potencia (Salazar, 2008; Maihold, 2011, p. 190).

\subsubsection{Una explicación alternativa: ¿'soft balancing'?}

Una explicación alternativa podría ser aquella desarrollada por Tolipov (2007) y Wehner (2011). ${ }^{5}$ Para estos autores, la diversificación es una estrategia de inserción internacional que sirve para hacer balance de poder. Incluso, Leslie Wehner (2011) la considera equivalente a una estrategia de soft balancing, que le ha permitido a Chile reducir su dependencia de esquemas de integración regional y poderes globales. Según este autor, los tratados de libre comercio constituyen una estrategia de diversificación de las exportaciones. Pero argumenta que no son tan solo eso. Más aún, Wehner (2011) considera los TLC como una herramienta de poder, ya que le han permitido a dicho país avanzar metas estratégicas en el ámbito político y económico, tales

\footnotetext{
5 Agradezco a los evaluadores por cuestionar hasta qué punto la estrategia de soft balancing se constituye en una explicación alternativa a la búsqueda de autonomía. Indudablemente, este es un importante tema de reflexión. Por ejemplo, el trabajo de Wehner (2011) concibe la diversificación económica como una plataforma que Chile ha desarrollado para hacer soft balancing a grandes poderes económicos globales y regionales, como lo son los Estados Unidos y el Mercosur. En este sentido, el concepto de soft balancing podría ser una explicación alternativa de los propósitos para los cuales se utiliza la diversificación, o, incluso, podría ser complementaria a aquella explicación que asocia la diversificación con la autonomía. No obstante, es esencial tener en cuenta que la utilidad y la aplicación de este concepto es actualmente un punto de debate en la literatura académica. El uso de este concepto que han hecho autores como Pape (2005), Paul (2004), Walt (2005), Art (2005) y Saltzmann (2012) ha sido cuestionado por ser teórica y metodológicamente defectuoso (Howorth y Menon, 2009), poco persuasivo (Lieber y Alexander, 2005) y que ha fracasado frente a explicaciones alternativas (Brooks y Wolforth, 2005). Además, es un concepto pensado como un mecanismo que podría surgir para balancear a los Estados Unidos con herramientas no militares, dada su supremacía militar a nivel mundial. Por lo tanto, sería necesario adaptar este concepto al contexto latinoamericano donde existe mayor capacidad militar relativa por parte de algunos actores, pero no supremacía global. En consecuencia, la construcción de una explicación alternativa que entienda la diversificación como instrumento para hacer soft balancing requiere mayor soporte académico y empírico. En contraste, el problema de la autonomía ha sido ampliamente discutido y documentado en la política exterior latinoamericana (Giacalone, 2012).
} 
como la reducción de la dependencia asimétrica en pocos mercados, influenciar polos de poder, mejorar las relaciones bilaterales y mejorar su estatus internacional.

También, es posible encontrar ejemplos de búsqueda de diversificación fuera de la región latinoamericana. Por su parte, Goh (2008) analiza el caso de países como Indonesia, Tailandia, Malasia, Singapur, Filipinas y Vietnam. Al respecto, muestra cómo dichos países han diseñado un modelo de inserción internacional que denomina omni-enmeshment. ${ }^{6}$ Para Goh (2008), estos países desean maximizar la limitada autonomía a la que pueden aspirar, debido a que son naciones pequeñas y dependientes de los países poderosos de la región. Por lo tanto, más que lograr la autonomía, buscan diversificar la dependencia en múltiples actores, como los Estados Unidos, Japón, China e India. En este contexto, experimentan una tensión entre el deseo por la autonomía y un pragmático reconocimiento de la inevitable dependencia.

Los países del Sudeste Asiático han adoptado una estrategia de diversificación amplia y multidireccional (Goh, 2008, p. 121). En el nivel regional, la Asean ha sido utilizada como el canal principal de vinculación con poderes extrarregionales, como Australia, Canadá, la Unión Europea, Japón, Nueva Zelanda, Rusia. Esto lo han hecho a través de instrumentos de diálogo político, foros regionales y la creación de normas regionales, como el Tratado de Amistad y Cooperación (TAC). El TAC se basa en el respeto a la mutua soberanía, a

\footnotetext{
6 Agradezco a los evaluadores por cuestionar hasta qué punto la estrategia omni-enmeshment es una estrategia de soft-balancing. Para Goh, el omni-enmeshment se basa en la creación de una compleja red de interconexión con el propósito de lograr la integración, la cual altera los intereses de los actores y posiblemente su identidad. Goh (2005a) señala que existen dos caminos para producir orden en el Sudeste Asiático: el omni-enmeshment y el balance de influencia. Una diferenciación entre estos dos conceptos es posible encontrarla en Goh (2005a). En el caso del Sudeste Asiático, según Goh (2005a), se ha dado un acercamiento entre países pequeños para hacer balance a China y no a los Estados Unidos, como lo sugeriría la teoría del balance de poder o el concepto de soft balancing. En relación con China, Goh (2005b) considera que el Sudeste Asiático ha combinado el omni-enmeshment como estrategia para acercarse a China con el soft balancing al involucrar a los Estados Unidos como balanceadores en la región, ver Goh (2005b).
} 
la integridad territorial, a la no interferencia en asuntos domésticos, a la renuncia al uso de la fuerza y a la solución pacífica de conflictos, que ha sido suscrito por Japón, Rusia, Corea del Sur, Australia y China con los países de la Asean (Goh, 2008). En el nivel bilateral, la estrategia de omni-enmeshment ha consistido en desarrollar relaciones más profundas en temas de seguridad.

En virtud de lo anterior, existen distintos autores que entienden la diversificación como un instrumento para hacer soft balancing $\mathrm{y} / \mathrm{u}$ omni-enmeshment. Estas se constituyen en explicaciones alternativas a aquella que nos ofrece la literatura sobre política exterior latinoamericana. ¿Cuál explicación es más adecuada? es una pregunta que debe ser desarrollada en términos empíricos.

\section{Conclusiones}

En este artículo, se muestra que existen diferentes concepciones sobre la noción de diversificación, la cual en algunos casos es asimilada como un instrumento de política pública; en otros, como un tipo de comportamiento internacional; y en otros, como un modelo de inserción internacional. Es importante destacar las variaciones en las definiciones como producto del lugar en el que emergen. Por ejemplo, en Asia Central obedece a preocupaciones de tipo político en materia de recursos naturales, en el Sudeste de Asia se plantea en relación con un mayor énfasis en la soberanía incorporando además de lo comercial una fuerte preocupación por asuntos políticos y de seguridad con otros países, mientras que en América Latina se define en relación con el objetivo de integrar aún más los esfuerzos políticos a los económicos.

En este texto, se ha mostrado la forma en que la diversificación tiene una relación estrecha con la autonomía, y cómo esta se convierte en el eje central o común que une la noción de diversificación en la política exterior latinoamericana. De igual manera, se muestra que la autonomía debe ser entendida como una variable fundamental que contribuye en gran medida a explicar la estrategia u orientación en el comportamiento internacional de un país débil. 
Por consiguiente, se ha insertado la discusión sobre la diversificación en un debate más amplio sobre la autonomía y la soberanía que preocupa esencialmente a países pequeños y medianos que son vulnerables y dependientes. Esto permite entender de forma comprensiva el papel e importancia de la diversificación como un instrumento que puede incidir de manera positiva en el acceso a la autonomía para reforzar los procesos de consolidación estatal. Se ha planteado que el tipo de autonomía que ejerce un país puede contribuir a revertir los efectos de una inserción internacional dependiente.

Esta diversidad de explicaciones nos deja frente a cierta ambigüedad y falta de claridad en cuanto a la relación existente entre atributos nacionales (tamaño, nivel de desarrollo, nivel de apertura política) y la posibilidad de llevar a cabo estrategias de diversificación, que vale la pena ser explorada en estudios futuros. En virtud de lo anterior, vale la pena subrayar la inexistencia de estudios comparados sobre este tema y la inexistencia de teorías de mediano alcance que tengan como propósito explicar los factores que producen la diversificación. De manera que sea posible ponderar o priorizar el nivel de influencia de los factores sistémicos o domésticos en la orientación y comportamiento internacional. Este ejercicio supera el alcance y propósito de este artículo.

En consecuencia, esta es un área donde existe espacio para futuras investigaciones. En primer lugar, aún es conveniente esclarecer de forma sistemática los factores que producen la diversificación, lo cual podría ser posible mediante la realización de ejercicios de comparación entre diferentes países y a través del estudio de períodos de tiempo más largos, a fin de lograr una mejor identificación de estos aspectos y un refinamiento a las aproximaciones existentes.

En segundo lugar, es importante teorizar sobre la relación entre el ejercicio de la autonomía y el comportamiento internacional de un país en proceso de consolidación estatal. Las especificidades de los países periféricos han puesto en constante evidencia las debilidades de enfoques teóricos que han sido construidos para explicar el comportamiento externo de países grandes y desarrollados. Para tal 
propósito, los estudios de caso pueden resultar de gran utilidad, ya que permitirían un desarrollo a mayor profundidad y enfocado exclusivamente en explorar los vínculos entre autonomía y diversificación, pues aún no es posible distinguir con certeza qué es primero.

En tercer lugar, el desarrollo de mecanismos de medición más sofisticados también puede contribuir en la comparación de patrones en la orientación de la política exterior de un país. Por último, otro aspecto aún inexplorado es el estudio de la alteración o redefinición de la identidad de la política exterior de un país a través de la ejecución de una estrategia de diversificación. Un mayor énfasis sobre 'cómo' se dan los procesos de diversificación y los efectos que va produciendo en el tiempo podría contribuir en esta dirección.

\section{Referencias}

Agosín, M. (2007). Export diversification and growth in emerging economies. Documentos de trabajo. Universidad de Chile, SDT 233. Recuperado de http://www.econ.uchile.cl/uploads/publicacion/7fec2632-b4c345a3-ab78-0970614f5bab.pdf

Ali, R., Alwang, J., \& Siegel, P. B. (1991). Is export diversification the best way to achieve export growth and stability? Working paper $\mathrm{N}^{\circ}$ 729. World Bank Policy Research and External Affairs. Recuperado de http://www. wds.worldbank.org/external/default/WDSContentServer/IW3P/ IB/1991/07/01/000009265_3961001182422/Rendered/PDF/ multi0page.pdf

Al-Marhubi, F. (2000). Export diversification and growth: an empirical investigation. Applied Economics Letters, 7(9), 559-562.

Amurgo-Pacheco, A., \& Pierola, M. D. (2008). Patterns of export diversification in developing countries: intensive and extensive margins. Working Paper Series $\mathrm{N}^{\mathrm{o}}$ 4473. World Bank Policy Research.

Art, R. J. (2005). Striking the Balance. International Security, 30(3), 177-85. doi:10.1162/isec.2005.30.3.177.

Ayoob, M. (1995). The third world security predicament: state making, regional conflict, and the international system. Boulder: Lynne Rienner Publishers. 
Barbosa, F., Posada, E., \& Serrano, E. (2011). La inserción de Colombia en Asia Pacifico. 2020: Colombia en el nuevo océano. Bogotá: Universidad Jorge Tadeo Lozano.

Baroni, P., \& Florencia, R. (2013). Relaciones entre América del Sur y China: ¿una alternativa para una inserción internacional autónoma? Documento de trabajo N ${ }^{\circ} 71$. Flacso.

Ben-Hammouda, H., Karingi, S. N., Njuguna, A. E., \& Sadni-Jallab, M. (2006) Diversification: towards a new paradigm for Africa's development. Work $\mathrm{N}^{\circ} 35$. United Nations, Economic Commission for Africa. Recuperado de http:/ / www.uneca.org/sites/default/files/publications/35.pdf

Berthelemy, J. C., \& Chauvin, S. (2000). Structural changes in Asia and growth prospects after the crisis. Working papers $\mathrm{N}^{\circ}$ 9. CEPII. Recuperado de http:/ /www.cepii.fr/PDF_PUB/wp/2000/wp2000-09.pdf

Blum, W. (2003). Killing hope: U.S. military and CLA interventions since World War II. Maine: Common Courage Press.

Brooks, S. G., \& Wohlforth, W. C. (2005). Hard times for soft balancing. International Security, (30), 72-108.

Buzan, B. (1991). People, States and Fear: An Agenda for international Security Studies in the Post-Cold War Era. Segunda Edición. Boulder, Colorado: Lynne Rienner Publishers.

Cardona, D. (2001). La política exterior de la administración Pastrana (19982002) hacia una evaluación preliminar. Colombia Internacional, (53), 53-74.

Cepeda, F. (1983). Comentario a la ponencia "El interés nacional y el ingreso a los No Alineados" de Marco Palacios. En M. Palacios (Ed.), Colombia no alineada. Bogotá: Biblioteca Banco Popular.

Drekonja, G. (1993). Autonomía periférica redefinida: América Latina en la década de los noventa. Documentos Ocasionales, (31).

Escudé, C. (1995). El realismo de los Estados débiles. Buenos Aires: GEL.

Faust, J., \& Franke, U. (2002). Attempts at diversification: Mexico and Pacific Asia. Pacific Review, 15(2), 299-324.

Faust, J., \& Mols, M. (1998). Latinoamérica y el Asia-Pacífico. Un acercamiento entre euforia y escepticismo. Revista Estudios Internacionales, 31(121-122), 10-35.

Faust, J. (2004). Latin America, Chile and East Asia: policy networks and successful diversification. Journal of Latin America Studies, 36(4), 743-770. 
Fernández de Soto, G., \& Pineda, S. (2012). El Pacifico: la inserción aplazada. La relación de Colombia con el Asia-Pacifico en el contexto de América Latina. Bogotá: Panamericana Formas e Impresos S.A.

Garza Elizondo, H. (1996). La política exterior de México: entre la dependencia y la diversificación. Foro Internacional, 36(4), 641-666.

Giacalone, R. (2012). "Latin American Foreign Policy Analysis: External Influences and Internal Circumstances: Latin American Foreign Policy Analysis". Foreign Policy Analysis 8(4), 335-54. doi:10.1111/j.17438594.2011.00176.x.

Goh, E. (2005a). Great powers and Southeast regional security strategies: omni-enmeshment, balancing and hierarchical order. Working paper $\mathrm{N}^{\mathrm{o}}$ 84. Singapur: Institute of Defence and Strategic Studies.

Goh, E. (2005b). Meeting the China challenge: the U.s. in Southeast Asian security regional strategies. Policy Studies, (16).

Goh, E. (2007-2008). Great powers and hierarchical order in Southeast Asia: analizing regional security strategies. International Security, (32), 113-157.

Goh, E. (2007). Southeast Asian reactions to America's new strategic imperatives. En J. D. Pollack (Ed.), Asia eyes America: regional perspectives on U.S. Asia-Pacific strategy in the 21t century. Newport: Naval War College.

Goh, E. (2008). Southeast Asia: strategic diversification in the 'Asian century'. En A. J. Tellis, M. Kuo \& A. Marble (Eds.), Strategic Asia 2008-09. Challenges and choices. Seattle: The National Bureau of Asian Research.

Gomes Saraiva, M. (2010). A diplomacia brasileira e as visões sobre a inserção externa do Brasil: institucionalistas pragmáticos x autonomistas. Mural Internacional, (1), 45-52.

Gutiérrez de Piñeres, S. A., \& Ferrantino, M. (1997). Export diversification and structural dynamics in the growth process: the case of Chile. Journal of Development Economics, 52(2), 375-391.

Herbst, J. (2000). States and power in Africa: comparative lessons in authority and control. Princeton: Princeton University Press.

Holsti, K. J. 1982. "Restructuring Foreign Policy: A Comparative Analysis". En K.J. Holsti et al. (Ed.). Why Nations Realign Foreign Policy Restructuring in the Postwar World. Winchester, MA: Allen and Unwin.

Howorth, J., \& Menon, A. (2009). Still Not Pushing Back: Why the European Union Is Not Balancing the United States. Journal of Conflict Resolution 53(5), 727-44. doi:10.1177/0022002709339362. 
Inayatullah, N. (1996). Beyond the Sovereignty Dilemma: Quasi-States as Social Construct. Cambridge Studies in International Relations, 46, 50-80.

Jackson, R. (1993). Quasi-States: sovereignty, international relations and the Third World. Cambridge: Cambridge University Press.

Jaguaribe, H. (abril-junio, 1979). Autonomía periférica y hegemonía céntrica. Estudios Internacionales, (46), 91-130.

Kahler, M. (December, 2000). The State of the State in world politics. Conference on the State of the Discipline, Washington, D.C.

Keohane, R. O. \& Nye, J. S. (1988). Poder e interdependencia: la politica mundial en transición. Buenos Aires: Grupo Editor Latinoamericano.

Lieber, K. A., \& Alexander, G. (2005). Waiting for balancing: why the world is not pushing.

Maihold, G. (2011). Reorientación y diversificación: América Latina entre nuevas oportunidades y viejos legados. En D. Wollrad, G. Maihold \& M. Mols (Eds.), La agenda internacional de América Latina: entre nuevas y viejas alianzas. Buenos Aires: Nueva Sociedad, Friedrich-Ebert-Stiftung, Wissenschaft und Politik. Recuperado de http://library.fes.de/pdffiles/nuso/08517.pdf

Mejía, J. F. (2011). Export diversification and economic growth: an analysis of Colombia's export competitiveness in the European Union's market". Berlin: Springer Verlag Heidelberg.

Moniz Bandeira, L. A. (2010). Presencia de los Estados Unidos en Brasil: dos siglos de historia. Buenos Aires: Editorial Corregidor.

Moon, B. (1983). The foreign policy of the dependent State. International Studies Quarterly, (27), 315-340.

Ocampo, J. A. (2008). Los paradigmas del desarrollo en la historia latinoamericana. En O. Altimir, E. V. Iglesias \& J. L. Machinea (Eds.), Hacia la revisión de los paradigmas de desarrollo en América Latina. Santiago de Chile: Comisión Económica para América Latina y el Caribe (Cepal).

Olano, A. (2012). Los cambios en la política exterior de Perú: diversificación, multilateralismo y nuevas alianzas estratégicas. En M. Ardila (Ed.), El Pacífico latinoamericano y su inserción internacional. Bogotá: Pontificia Universidad Javeriana.

Olivet, M. C. (2005). Unravelling interregionalism theory: a critical analysis of the new relations between Latin America and East Asia. Ponencia presentada en la VI Reunión de la Red de Estudios de América Latina y el Caribe 
sobre Asia-Pacífico (Redealap), Banco Interamericano de Desarrollo. Recuperado de www.iadb.org/intal/aplicaciones/uploads/ponencias/ Foro_REDEALAP_2005_16_Olivet.pdf

Pape, R. A. (2005). Soft balancing against the United States. International Security, (30), 7-45.

Pardo, R., \& Tokatlian, J. (1988). Política exterior colombiana. ¿De la subordinación a la autonomía? Bogotá: Tercer Mundo Editores, Uniandes.

Pardo, R. (2001). Relaciones internacionales y proceso de paz. Proyecciones sobre escenarios. Colombia Internacional, (51), 28-50.

Paul, T. V. (2004). Soft balancing in the age of U.S. primacy. International Security, (30), 46-71.

Pollock, D., Kerner, D. \& Love, J. (2001). Entrevista Inédita a Prébisch: Logros y Deficiencias de La CEPAL. Revista de La CEPAL, 75, 9-23.

Protsenko, A. (2013). México: diversificación de la política exterior hacia Asia. Iberoamerica, (1), 57-80.

Puig, J. C. (1979). Doctrinas internacionales y autonomia latinoamericana. Caracas: Universidad Simón Bolívar.

Richardson, N., \& Kegley, C. Trade dependence and foreign policy compliance: longitudinal analysis. International Studies Quarterly, (24), 191-222.

Rodrik, D. (2005). Políticas de diversificación económica. Revista Cepal, (87), 7-23.

Rubiolo, F. (2011). La relación comercial entre Argentina y Singapur: perspectivas y oportunidades para la provincia de Córdoba. En G. Santillán, N. Mohaded, H. Moreno \& M. F. Rubiolo (Eds.), América Latina y el Este Asiático: perspectivas desde Córdoba (pp. 177-190). Córdoba: Centro de Investigación y Estudios de la Cultura y la Sociedad (CIECS).

Rubiolo, M. F. (2012). Relaciones Argentina-Sudeste de Asia: vínculos comerciales y políticos contemporáneos (2003-2011). En C. Morasso \& G. Pereyra (Eds.), Argentinay Brasil:proyecciones internacionales, cooperación sur-sure integración. Rosario: UNR Editora. Recuperado de http://www. catedrarrii.com.ar/docs/libro-Cooperacion-SUR-SUR.pdf

Russell, R., \& Tokatlian, J. (2010). Autonomía y neutralidad en la globalización. Buenos Aires: Capital Intelectual.

Sánchez, P. (2003). Panama: a ‘hegemonized’ foreign policy. En J. Hey (Ed.), Small states in world politics. Boulder: Lynne Rienner Publishers.

Salazar, R. (2008). La política exterior de Hugo Chávez. Revista Mexicana de Política Exterior, (83), 221-254. 
Saltzman, I. (2012). Soft balancing as foreign policy: assessing American strategy toward Japan in the interwar period. Foreign Policy Analysis, $8(2), 131-150$.

Samen, S. (2010). A primer on export diversification: key concepts, theoretical underpinnings and empirical evidence. Working paper. World Bank Institute. Recuperado de https:/ / blogs.worldbank.org/files/growth/EXPORT_ DIVERSIFICATION_A_PRIMER_May2010(1).pdf

Thies, C. (2014). Role theory and foreign policy analysis in Latin America. Foreign Policy Analysis, (0), 1-20.

Tickner, A. (2002). Los estudios internacionales en América Latina. ¿Subordinación intelectual o pensamiento emancipatorio? Bogotá: Alfaomega y Uniandes.

Tickner, A. (2003). Seeing IR differently: notes form the Third World. Journal of International Studies, (32), 295-324.

Tokatlian, J. (1993). Autonomía y política exterior: un debate abierto, un futuro incierto. Documentos Ocasionales, (31), 22-38.

Tokatlian, J. (1996). Posguerra Fría y política exterior: de la autonomía relativa a la autonomía ambigua. Revista Análisis Político, (28), 25-48.

Tokatlian, J. (2011). Latinoamérica y sus 'alianzas' extrarregionales: entre el espejismo, la ilusión y la evidencia. En D. Wolrad et al. (Eds.), La agenda internacional de América Latina: entre nuevas y viejas alianzas. Buenos Aires: FES-Fundación Nueva Sociedad. Recuperado de http:/ / library. fes.de/pdf-files/nuso/08517.pdf

Tolipov, F. (2007). The foreign policy orientations of Central Asian States: positive and negative diversification. Acta Slavica laponica, (16), 23-40. Recuperado de http://www.isn.ethz.ch/isn/DigitalLibrary/Publications /Detail/?ots591=0c54e3b3-1e9c-be1e-2c24a6a8c7060233\&lng=en\&id $=58351$

Vargas, L. F., Sosa, S., \& Rodríguez Ríos, J. D. (2012). El comercio como plataforma de la política exterior colombiana en la administración de Juan Manuel Santos. Colombia Internacional, (76), 259-292.

Vigevani, T., Fernandes de Oliveira, M., \& Simpson, T. (2007). Brazilian foreign policy in the Cardoso era: the search for autonomy trough integration. Latin American Perspectives, (34), 57-80.

Vigevani, T., \& Cepaluni, G. (2007). Lula's foreign policy and the quest for autonomy through diversification. Third World Quarterly, 28(7), 1309-1326.

Walt, S. (2005). Taming American Power. Foreign Affairs, 84(5). 


\section{I FABIO FORERO}

Wehner, L. (2011). Chile's rush to free trade agreements. Revista de Ciencia Política, 31(2).

Wilhelmy, M., \& Lazo, R. M. (1997). La estrategia bilateral de Chile en el Sudeste Asiático. Revista Ciencia Política, (19), 37-60. 